Military Technical College

Kobry El-Kobbah, Cairo, Egypt

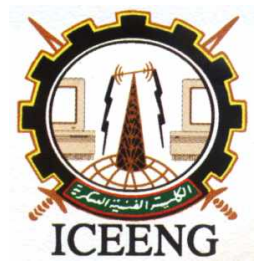

$6^{\text {th }}$ International Conference

on Electrical Engineering

ICEENG 2008

\title{
Estimation of multipath parameters for microwave line of sight links
}

\begin{abstract}
By
Mohamed A H Eleiwa (Ph.D.)

Egyptian Armed Forces

\section{$\underline{\text { Abstract }}$}

On the basis of graphical ray tracing along with the link geometry and configuration, this paper develops a simplified graphical - numerical technique to estimate the multipathing parameters associated with the multiple paths resulting from a given vertical refractivity profile of a two-layer troposphere. The proposed model is verified by applying it to analyze the performance of different microwave line of sight (MLOS) links with different parameters and configurations. The multiple-path rays are displayed over each MLOS link with a given geometry and configuration. The numerical results are discussed and compared with the corresponding published data using different techniques. The results show clearly the dependence of multipath parameters on refractive index gradient, link geometry and link configuration.
\end{abstract}

\section{$\underline{\text { I. Introduction }}$}

Terrestrial propagation of microwaves is significantly affected by tropospheric refraction caused by irregular and changing nature of the troposphere. Such tropospheric inhomogenities produce the refractive multipath fading. Fading is the continuous and rapid variations in radio field strength occurring randomly at the point of reception, thereby producing changes in the signal level, and introducing distortion. Due to inhomogeneities in the troposphere, propagating rays encounter variations in refractive index that cause refractive multipathing. For narrow-band signals, multipath causes signal loss due to flat fading. As the relative bandwidth increases, each frequency component in the signal is attenuated differently causing in-band distortion, which is called selective fading. Multipath fading also affects systems using a dual-polarized ratio channel by reducing the cross polarization discrimination. Multipath fading is therefore considered the major source of signal fading and 
consequently as the main factor contributing to the breakdown of MLOS systems.

In order to assess the consequences of multipath fading effects on MLOS links, various experimental $[1,2]$ and theoretical models $[3,4,5,6]$ have been used to estimate multipathing parameters. The simplifying assumptions about link geometry in [3] limit its utility. Complex quartic ray equations have been derived and solved in $[4,5,6]$ to analyze multipath propagation phenomena over each link configuration. A ray-tracing approach has been presented in [7] to provide the multipath rays. An attempt to estimate the effects of receiving angle variations on MLOS systems was presented in [8].

Apart from the mathematical difficulties arising from the solutions of the complicated ray equations for every link configuration, this paper develops a generalized numerical technique based on a graphical ray plotting approach. The two-layer tropospheric model with an elevated duct is first described in section II. The ray plotting technique is explained in section III, and the multipathing parameters are then derived. To demonstrate the dependence of discrete path parameters on link geometry and configuration, numerical calculations and discussions are presented in section IV.

\section{Two-Layer Tropospheric Model}

The analysis of multipath propagation is performed assuming a simplified two-layer tropospheric model with an elevated inversion layer (gradient steeper than $-157 \mathrm{Nu} / \mathrm{km}$ ), and the lower layer is usually a standard troposphere (gradient $=-40 \mathrm{Nu} / \mathrm{km}$ ), as shown in Fig. (1.a). The refractive index $n$ is assumed to vary only in the vertical direction $\mathrm{z}$ with no horizontal variation according to the following relation [9].

$$
\frac{\mathrm{dN}}{\mathrm{dz}}=77.6\left[\frac{1}{\mathrm{~T}} \frac{\mathrm{dp}}{\mathrm{dz}}-\left(\frac{\mathrm{p}}{\mathrm{T}^{2}}+\frac{9620 \mathrm{e}}{\mathrm{T}^{3}}\right) \frac{\mathrm{dT}}{\mathrm{dz}}+\frac{4810}{\mathrm{~T}^{2}} \frac{\mathrm{de}}{\mathrm{dz}}\right] \mathrm{m}^{-1}
$$

Where $\boldsymbol{T}$ is the temperature in Kelvin, $\boldsymbol{p}$ is the pressure, $\boldsymbol{e}$ is the humidity in millibars, and $N=(\boldsymbol{n}-1) 10^{6}$ is the excess index of refraction. The modified refractive index $\boldsymbol{m}$ with a flattened earth's model is used to account for the curvature of the earth.

In the standard troposphere region $\left(z<\boldsymbol{h}_{L}\right), \boldsymbol{m}(z)$ has a positive gradient equal to $\mathbf{1 / R _ { f }}$ upwards. Thus

$$
\boldsymbol{m}(z)=\boldsymbol{m}_{o}+z / \boldsymbol{R}_{f}
$$

Where $\boldsymbol{m}_{\boldsymbol{o}}$ is a reference level modified refractive index. Upon entering the inversion layer at height $\boldsymbol{h}_{L}$, the curvature is $\mathbf{l} \boldsymbol{R}$ downwards. Thus the modified refractive index applicable to the inversion layer $\left(z>h_{L}\right)$ is given by $m_{L}(z)=m_{o}+h_{L} / R_{f}+(-1 / R)\left(z-h_{L}\right)$

The radius of curvature $\boldsymbol{R}$ of the path is a function of the rate of change of the dielectric constant $\varepsilon_{r}$ with height $z$. Thus 


$$
\begin{aligned}
& R=-1 /(\partial m / \partial z) \\
& m(z)=n(z)+z / a
\end{aligned}
$$

Where $n(z)=\sqrt{\varepsilon_{r}(z)}$, and $\boldsymbol{a}=6.73 \times 10^{6} \mathrm{~m}$ is the earth's radius.

Once the refractivity profile is obtained for the assumed two-layer tropospheric model as described by the above equations, the desired multipathing parameters for a given link geometry may be determined by using an accurate graphicalnumerical technique, which is explained in the following section.

\section{The Multipathing Graphical-Numerical Technique}

For a communication link, the voltage produced by the direct wave at the receiving antenna is approximately given by [8] as $V_{r e c} \propto f_{1}\left(\theta_{t}\right) f_{2}\left(\theta_{r}\right) \frac{e^{-j k r}}{4 \pi r}$

Where $\boldsymbol{r}$ is the ray path length, $f_{1}$ and $f_{2}$ are the radiation field strength patterns of the transmitting and receiving antennas. For multipath propagation conditions shown in Fig. (1.b), the resultant received signal is due to different rays with different path lengths producing either constructive or destructive interference at the receiving point.

As an example, consider the link configuration shown in Fig. (1.b), where both transmitting and receiving antennas are below the inversion layer interface with $\boldsymbol{h}_{r}>\boldsymbol{h}_{t}$. The path length $\boldsymbol{r}_{\boldsymbol{n}}$ of the $\boldsymbol{n}^{\text {th }}$ ray travelling from the transmitter to the receiver is derived as $\boldsymbol{r}_{n}=\boldsymbol{R}_{f}\left(\theta_{t_{n}}+\theta_{r_{n}}\right)+2 n \boldsymbol{R} \theta_{b_{n}}+\mathbf{2}(\boldsymbol{n}-1) \boldsymbol{R}_{f} \theta_{b_{n}}+\boldsymbol{R}_{f}\left(\theta_{b_{n}}+\theta_{r_{n}}\right)$

Where $\boldsymbol{n}$ is the number of ray segments within the inversion layer. The transmitting (launching), crossing, and receiving (AOA) angles for the $\theta \mathrm{n}^{\text {th }}$ ray are denoted by $\theta_{t_{n}}, \theta_{b_{n}}$, and $\theta_{r_{n}}$; respectively. The path length of the direct ray $\boldsymbol{r}_{\boldsymbol{o}}$, which suffers no refraction from transmitting to receiving antennas, is obtained by substituting $\boldsymbol{n}=\mathbf{0}$ into (7) to get

$\boldsymbol{r}_{\boldsymbol{o}}=\boldsymbol{R}_{f}\left(\theta_{t o}+\theta_{r o}\right)$

Varying the launching angle $\theta_{t}$ within the transmitting antenna beamwidth, the resulting crossing and receiving angles, which comply with the given link geometry and configuration in Fig.1, are derived as

$$
\begin{gathered}
\theta_{b}=\cos ^{-1}\left(\cos \theta_{t}-\frac{h_{L}-h_{t}}{R_{f}}\right) \\
\theta_{r}=\cos ^{-1}\left(\cos \theta_{b}-\frac{h_{L}-h_{r}}{R_{f}}\right)
\end{gathered}
$$

Assuming ray transmission and reception are only within the antenna beamwidth, which results in very small launch and receive angles, and hence antenna patterns may be considered isotropic. Substituting the values of $r_{n}$ and $r_{o}$ 
obtained from (7) and (8); respectively, into (6) to give the relative complex amplitude $\alpha_{n}$ as

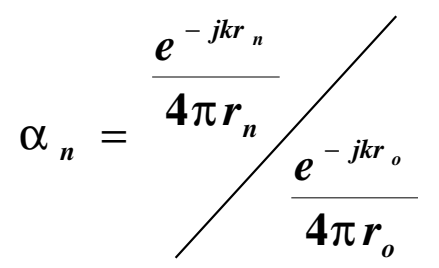

and the relative time delay $\tau_{n}$ is also obtained as

$$
\tau_{n}=\left(\boldsymbol{r}_{n}-\boldsymbol{r}_{o}\right) / \boldsymbol{c}
$$

Similarly, the proposed procedures for obtaining the multipathing parameters $\theta_{r}, \tau$, and $\alpha$ are based on incrementing the launching angle $\theta_{t}$ within the specified beamwidth to produce several rays with different path parameters. The crossing and receiving angles are then calculated for the rays contributing to the received signal. The parameters of such rays should also satisfy the prescribed link geometry and configuration. Each ray path is then plotted as circular arc segments starting from the transmitter with known launching angle $\theta_{t n}$, crossing the boundary $\mathrm{n}$ times at $\theta_{b n}$, and then hitting the receiving antenna at the receiving angle (angle of arrival) $\theta_{r n}$.

Finally, the ray path length $r_{n}$ is estimated as the summation of its constituting ray segments, and consequently the relative amplitude and time delay are calculated using equations (11) and (12).

\section{Numerical calculations and discussion}

Numerical calculations are performed in this section to study the variation of multipath ray parameters (launching angle $\theta_{t}$, and angle of arrival $\theta_{r}$ ) with varying link parameters for several link configurations (transmitting antenna height $\boldsymbol{h}_{\boldsymbol{t}}$, inversion layer height $\boldsymbol{h}_{\boldsymbol{L}}$, receiving antenna height $\boldsymbol{h}_{\boldsymbol{r}}$, ground link distance $\boldsymbol{L}$, and inversion layer refractivity gradient IRG).

Fig.2 illustrates the variation of $\theta_{r}$ for quasi direct rays $(\boldsymbol{n}=\mathbf{1})$ versus $\boldsymbol{h}_{\boldsymbol{L}}$, while $\theta_{t}$ is incremented within the $3 \boldsymbol{d} \boldsymbol{B}$ antenna beamwidth $\left(\approx \pm \boldsymbol{0 . 2 ^ { \circ }}\right)$, for a microwave LOS link with $h_{t}=85 \mathrm{~m}, h_{r}=125 \mathrm{~m}, \mathbf{L}=100 \mathrm{~km}, I R G=-30 o N u / \mathrm{km}$, and the ground refractivity gradient $\boldsymbol{G R G = - 0 N u / k m}$. Fig.2 shows clearly the coincidence between the calculated and published results by [6]. The effect of varying the link length $\boldsymbol{L}$ from $20 \mathbf{~} \mathbf{m}$ to $200 \mathbf{~} \mathbf{m}$ on the values of the refracted rays angles $\theta_{t}, \theta_{b}$, and $\theta_{r}$, is studied and plotted in Fig.3 for only first order refracted rays $(n=1)$, over a link with $h_{t}=100 m, h_{r}=105 m . h_{L}=110 m$, and $I R G=-$ $300 \mathrm{Nu} / \mathrm{km}$. It is always noted that $\left|\theta_{b}\right|>\left|\theta_{r}\right|$ and $\left|\theta_{b}\right|>\left|\theta_{t}\right|$. It is also noted that no refracted rays are detected for $\boldsymbol{L}<\mathbf{4 0 \mathbf { k m }}$, while higher-order refracted rays $(\boldsymbol{n}>\mathbf{2})$ are detected for $\boldsymbol{L}>\mathbf{1 2 0} \mathbf{k m}$. Higher order rays are found to have shallower angles than lower-order rays, as in the case of $\boldsymbol{L}=\mathbf{1 4 0} \mathbf{k m}$, where : 
$\theta_{t 2}=\mathbf{0 . 0 6 3 4}^{o}<\theta_{t 1}=\mathbf{0 . 1 4 8 9}^{\circ}, \theta_{b 2}=\mathbf{0 . 1 0 8 1}^{o}<\theta_{b 1}=\mathbf{0 . 1 7 2 7}^{\circ}, \theta_{r 2}=\mathbf{0 . 0 8 8 6}^{o}<\theta_{r 1}=\mathbf{0 . 1 6 1 2}^{o}$

The receiving angle variation $\theta_{r}$ with the transmitting antenna height $h_{t}$ is also studied and shown in Fig.4 for the same MLOS link parameters of Fig.3, but with $\mathbf{L}=100 \mathbf{k m}$, and variable $\boldsymbol{h}_{\boldsymbol{t}}$. It is noted that multipath occurs only when the height difference between the terminals is small enough; namely, $\boldsymbol{h}_{\boldsymbol{r}} \boldsymbol{-} \boldsymbol{h}_{\boldsymbol{t}}<\mathbf{2 0 \boldsymbol { m }}$. The effect of varying the inversion layer refractivity gradient $\boldsymbol{I} \boldsymbol{R} \boldsymbol{G}$ on the refracted rays angles is also investigated for the above link at $h_{t}=100 \mathrm{~m}$. The results are plotted in Fig.5 for only quasi-direct rays $(n=1)$. It is noted that multipath occurs only when $|\boldsymbol{I R G}|-|\boldsymbol{G R G}|>\mathbf{2 0 0} \mathbf{N u / \mathbf { k m }}$, and higher-order rays are detected for $|I R G|>350 \mathrm{Nu} / \mathbf{k m}$.

Knowing the angles of the multipath rays, the path lengths $\boldsymbol{r}_{\boldsymbol{n}}$ and $\boldsymbol{r}_{\boldsymbol{o}}$ are first determined according to (7) and (8), and then by substituting the obtained path lengths into (11) and (12) to get the relative amplitudes and delays. As an example, the relative delays of the first-order $(n=1)$ refracted rays are calculated for a link with $h_{t}=85 \mathrm{~m}, h_{r}=125 \mathrm{~m}, L=100 \mathrm{~km}, I R G=-300 \mathrm{Nu} / \mathrm{km}, G R G=-$ $40 \mathrm{Nu} / \mathrm{km}$, and variable inversion layer height $\boldsymbol{h}_{\boldsymbol{L}}$. There are a slight discrepancy between the calculated results and those published in [6] as shown in Fig.6.

Finally the multipath problem is wholly represented by accurately tracing the different rays launched from the transmitting antenna and possibly hitting the reception point for different link geometry and configuration. Fig.7 shows two different rays with $\boldsymbol{n}=\boldsymbol{l}$ and $\boldsymbol{n}=2$, launched at $\theta_{t 1}=\mathbf{0 . 1 6 3 9}^{\circ}, \theta_{t 2}=\mathbf{0 . 0 1 2 6}^{\circ}$, crossing the inversion layer interface at $\boldsymbol{h}_{L}=\mathbf{1 0 0} \boldsymbol{m}$ with $\theta_{b 1}=\mathbf{0 . 1 9 5 9}^{\circ}, \theta_{b 2}=\mathbf{0 . 1 0 8}^{\circ}$, and hitting the receiving antenna at height $\boldsymbol{h}_{r}=105 \mathrm{~m}$, which exists in the duct with $I R G=-300 \mathbf{N u} / \mathbf{k m}$, and at a ground distance $\boldsymbol{L}=\mathbf{1 0 0} \mathbf{k m}$ from the transmitting antenna. The obtained receiving angles are $\theta_{r 1}=\mathbf{0 . 1 8 3 5}^{\circ}, \theta_{r 2}=\mathbf{0 . 0 8 3 5}^{\circ}$. The direct ray, which would exist if there were no ducting region, is also plotted for comparison with the refracted rays in the same figure. Another multipath problem is investigated for a different link configuration $\left(\boldsymbol{h}_{\boldsymbol{L}}>\boldsymbol{h}_{\boldsymbol{r}}>\boldsymbol{h}_{t}\right)$, and depicted in Fig.8. It is clear from Fig.7 and Fig.8 that the link communication is performed through the refracted rays only. Direct ray communication is impossible for the link configuration shown in Fig.7, while the whole configuration of Fig.8 should be $110 \mathrm{~m}$ higher in order to avoid the direct ray reflections from the ground plane, and hence direct ray communication may be possible.

\section{V-Conclusion}

The developed model in this paper is based on incrementing the launching angle $\theta_{t}$ within the antenna beamwidth, and then looking for the combination of $\theta_{t}, \theta_{b}$, and $\theta_{r}$, which satisfies a given link geometry and configuration in a twolayer tropospheric region. The proposed model has been applied to investigate the variation of multipathing parameters with geometrical link parameters for different link configurations. The refractive multipathing phenomena has then 
been analyzed and represented graphically. It is suggested that, a careful study for the outcome of the numerical and the graphical results, will help to choose the suitable link parameters and configuration, which satisfy minimum signal fading due to refractive multipathing phenomena.

\section{$\underline{\text { References }}$}

[1] A. R. Lowry, C. Rocken, and S. V. Sokolovskiy, "Vertical profiling of atmospheric refractivity ground-based GPS," Radio Science, Vol. 37, No. 3, 10.1029/2000RS002565, 2002.

[2] G. Beyrele, K. Hocke, J. Wickert, T. Schmidt, C. Marquardt, and C. Reigber, "GPS radio occulation with CHAMP; A radio holographic analysis of GPS signal propagation in the troposphere and surface reflections," Journal of geophysical research, Vol. 107, No. D24, 4802, doci: 10.1029/2001JD001402, 2002.

[3] C.L.Ruthroff,"Multiple-path fading on line-of-sight microwave radio systems as a function of path length and frequency," BSTJ, Vol. 50, pp.23752398, September 1971.

[4] L. W. Pickering, and J. Derosa, "Refractive multipath model for line-of-sight microwave relay links," IEEE trans. on Communications, Vol. COM-27, No.8, August 1979.

[5] S. F. Mahmoud, H. N. Boghdadi, and O.L. El-Sayed, "A theoretical model for multipath fading on line of sight propagation," $3^{\text {rd }}$ NRSC, pp.320-334, Cairo, Egypt, Feb. 1985.

[6] Steen A. Parl, "Characterization of multipath parameters for line-of-sight microwave propagation," IEEE trans. on A\&P, vol. AP-31, no. 6, Nov. 1983

[7] Alan R. Webster, "Ray path parameters in tropospheric multipath propagation,” IEEE trans. on A\&P, vol. AP-30, no. 4, July1982.

[8] Alan R. Webster, "Angle of arrival considerations in microwave communications systems," IEEE trans. on A\&P, vol. AP-30, no.4, July 1982.

[9] K. D. Prasad, "Antenna and wave propagation," Tech. India publications, 16/7698, NewDelhi-110005, 1997. 


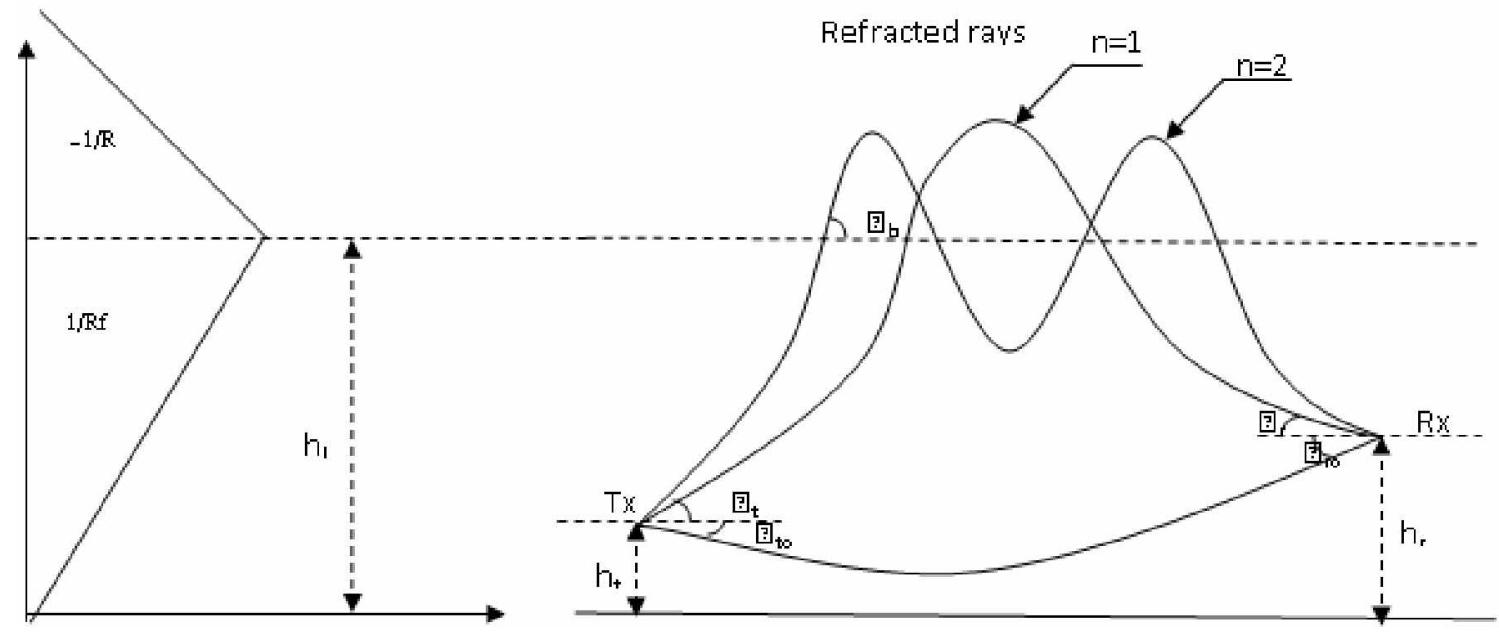

(a) Modified refractive index profile. (b) Link geometry and multipath parameters. Fig.(1) Link geometry and ray parameters for a refractivity profile of a two-layer tropospheric model.

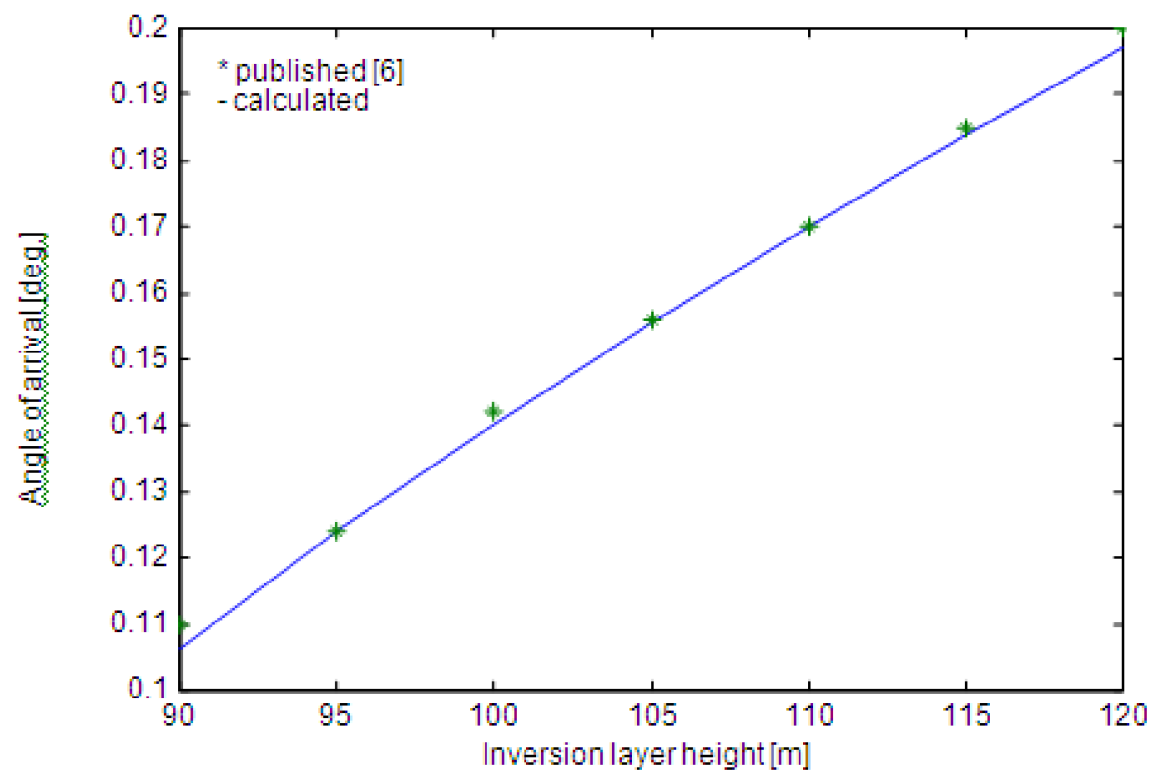

Fig.(2) Receiving angle variations for quasi-direct rays vs. inversion layer height. 


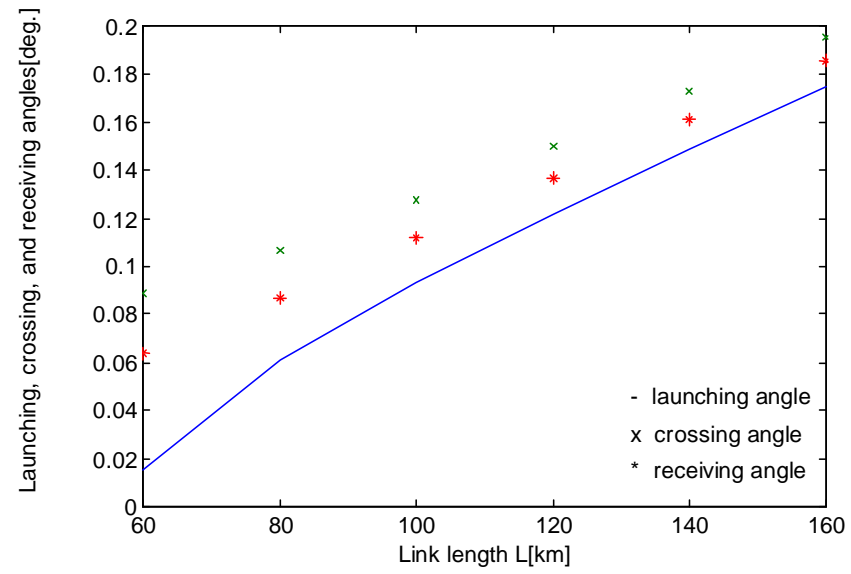

Fig.(3) Variation of launching, crossing, and receiving angles for first-order refracted rays $(n=1)$ vs. link distance $L$.

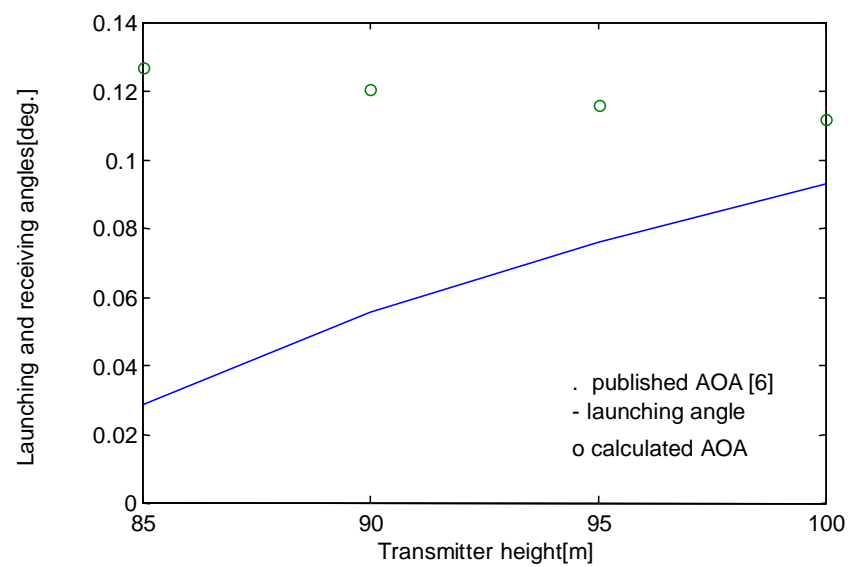

Fig.(4) Launching angles, calculated and the available published receiving angles for first-order refracted rays, as functions of $h_{t}$.

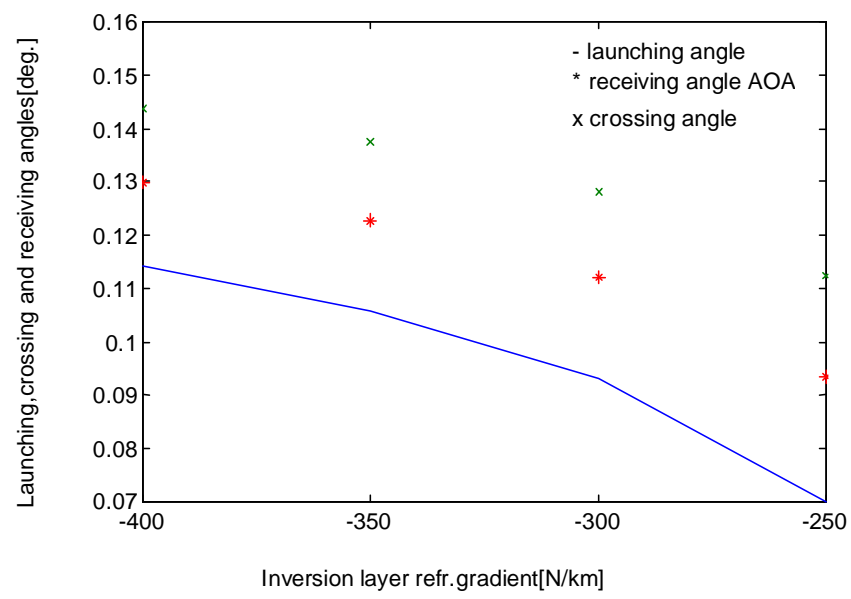

Fig.(5) The variation of first-order refracted rays angles vs. IRG 


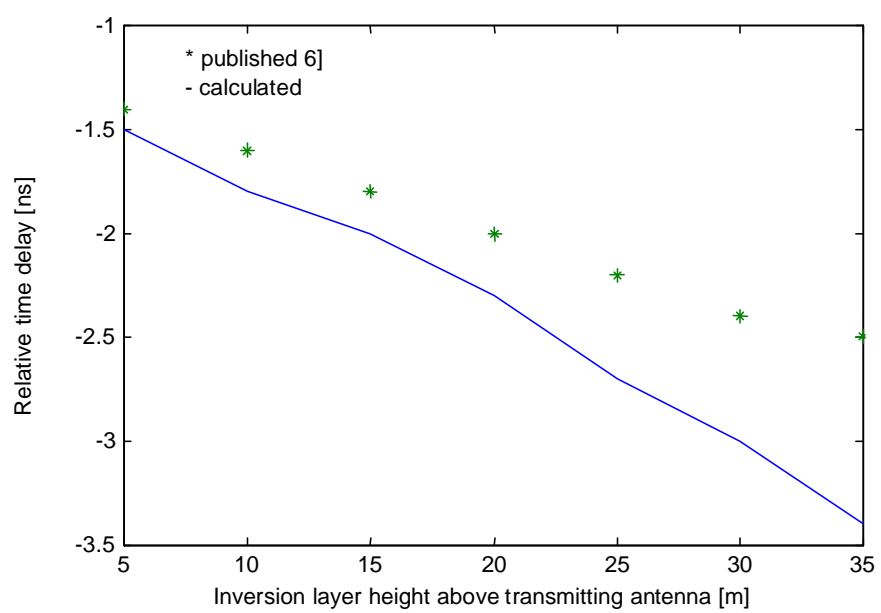

Fig.(6) Calculated and published relative time delay for quasi-direct rays vs. the height difference $h_{L}-h_{t}$.

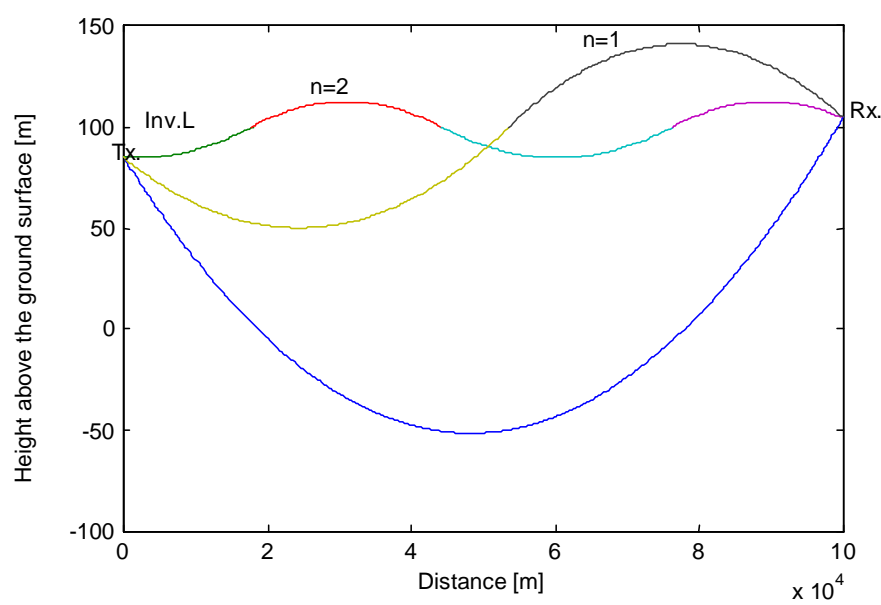

Fig.(7) Refracted rays for a specified link with $h_{r}>h_{L}>h_{t}$.

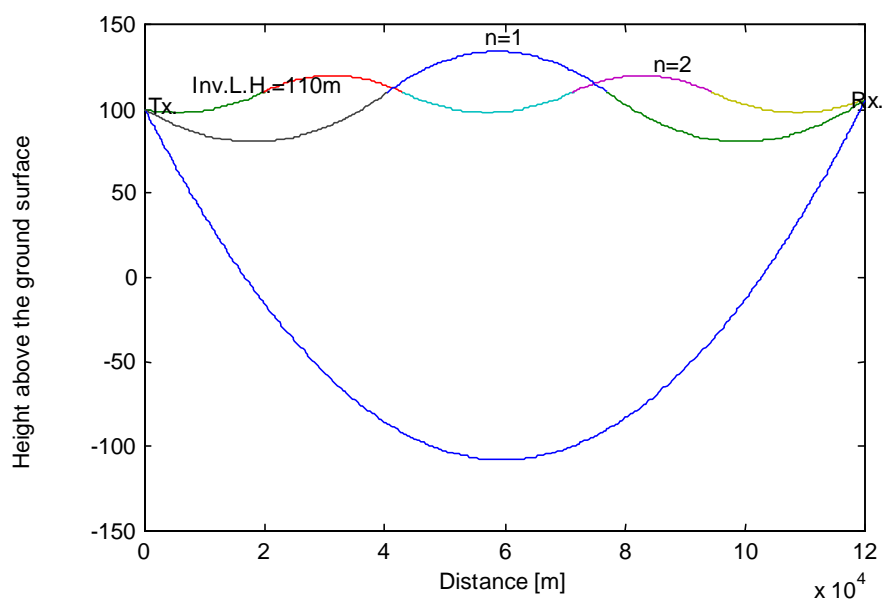

Fig.(8) Refracted rays for a specified link with $h_{L}>h_{r}>h_{t}$. 